\title{
REAL HYPERSURFACES IN COMPLEX TWO-PLANE GRASSMANNIANS WHOSE SHAPE OPERATOR IS OF CODAZZI TYPE IN GENERALIZED TANAKA-WEBSTER CONNECTION
}

\author{
Kyusuk Cho, Hyunjin Lee, and Eunmi PaK
}

\begin{abstract}
In this paper, we give a non-existence theorem of Hopf hypersurfaces in complex two-plane Grassmannians $G_{2}\left(\mathbb{C}^{m+2}\right), m \geq 3$, whose shape operator is of Codazzi type in generalized Tanaka-Webster connection $\hat{\nabla}^{(k)}$
\end{abstract}

\section{Introduction}

Let us denote by $G_{2}\left(\mathbb{C}^{m+2}\right)$ the set of all complex two-dimensional linear subspaces in $\mathbb{C}^{m+2}$, which is said to be a complex two-plane Grassmannian. This Riemannian symmetric space $G_{2}\left(\mathbb{C}^{m+2}\right)$ has a remarkable geometric structure. It is the unique compact irreducible Riemannian manifold being equipped with both a Kähler structure $J$ and a quaternionic Kähler structure $\mathfrak{J}$ not containing $J$, for details we refer to [2], [3] and [4]. In particular, when $m=1, G_{2}\left(\mathbb{C}^{3}\right)$ is isometric to the two-dimensional complex projective space $\mathbb{C} P^{2}$ with constant holomorphic sectional curvature eight. When $m=2$, we note that the isomorphism $\operatorname{Spin}(6) \simeq \mathrm{SU}(4)$ yields an isometry between $G_{2}\left(\mathbb{C}^{4}\right)$ and the real Grassmann manifold $G_{2}^{+}\left(\mathbb{R}^{6}\right)$ of oriented two-dimensional linear subspaces in $\mathbb{R}^{6}$. In this paper, we will assume $m \geq 3$.

Moreover, naturally we could consider two geometric conditions for hypersurfaces $M$ in $G_{2}\left(\mathbb{C}^{m+2}\right)$ that the 1-dimensional distribution $[\xi]=\operatorname{Span}\{\xi\}$ and the 3 -dimensional distribution $\mathfrak{D}^{\perp}=\operatorname{Span}\left\{\xi_{1}, \xi_{2}, \xi_{3}\right\}$ are both invariant under the shape operator $A$ of $M$ (see Berndt and Suh [3]). Here the almost contact structure vector field $\xi$ defined by $\xi=-J N$ is said to be a Reeb vector field, where $N$ denotes a local unit normal vector field of $M$ in $G_{2}\left(\mathbb{C}^{m+2}\right)$. The almost contact 3 -structure vector fields $\xi_{\nu}$ for the 3-dimensional distribution

Received July 30, 2012; Revised June 18, 2013.

2010 Mathematics Subject Classification. Primary 53C40; Secondary 53C15.

Key words and phrases. complex two-plane Grassmannians, Hopf hypersurface, generalized Tanaka-Webster connection, shape operator, Codazzi type tensor.

The second author was supported by NRF grants No. 2012-R1A1A3002031 and No. 20110030044 (SRC-GAIA). 
$\mathfrak{D}^{\perp}$ of $M$ in $G_{2}\left(\mathbb{C}^{m+2}\right)$ are defined by $\xi_{\nu}=-J_{\nu} N(\nu=1,2,3)$, where $J_{\nu}$ denotes a canonical local basis of a quaternionic Kähler structure $\mathfrak{J}$, such that $T_{x} M=\mathfrak{D} \oplus \mathfrak{D}^{\perp}, x \in M$

By using such two geometric conditions and the results in Alekseevskii [1], Berndt and Suh [3] proved the following:

Theorem A. Let $M$ be a connected real hypersurface in $G_{2}\left(\mathbb{C}^{m+2}\right), m \geq 3$. Then both $[\xi]$ and $\mathfrak{D}^{\perp}$ are invariant under the shape operator of $M$ if and only if

(A) $M$ is an open part of a tube around a totally geodesic $G_{2}\left(\mathbb{C}^{m+1}\right)$ in $G_{2}\left(\mathbb{C}^{m+2}\right)$, or

(B) $m$ is even, say $m=2 n$, and $M$ is an open part of a tube around a totally geodesic $\mathbb{H} P^{n}$ in $G_{2}\left(\mathbb{C}^{m+2}\right)$.

When the Reeb flow on $M$ in $G_{2}\left(\mathbb{C}^{m+2}\right)$ is isometric, we say that the Reeb vector field $\xi$ on $M$ is Killing. This means that the metric tensor $g$ is invariant under the Reeb flow of $\xi$ on $M$. In [4], Berndt and Suh showed that this notion is equivalent that the shape operator $A$ commutes with the structure tensor $\phi$. From this, they also gave a characterization of real hypersurfaces of type (A) in Theorem $\mathrm{A}$ in terms of the Reeb flow on $M$ as follows (see [4]) :

Theorem B. Let $M$ be a connected orientable real hypersurface in $G_{2}\left(\mathbb{C}^{m+2}\right)$, $m \geq 3$. Then the Reeb flow on $M$ is isometric if and only if $M$ is an open part of a tube around a totally geodesic $G_{2}\left(\mathbb{C}^{m+1}\right)$ in $G_{2}\left(\mathbb{C}^{m+2}\right)$.

On the other hand, Lee and Suh [11] gave a new characterization of real hypersurfaces of type $(\mathrm{B})$ in $G_{2}\left(\mathbb{C}^{m+2}\right)$.

Theorem C. Let $M$ be a connected orientable Hopf real hypersurface in $G_{2}\left(\mathbb{C}^{m+2}\right), m \geq 3$. Then the Reeb vector field $\xi$ belongs to the distribution $\mathfrak{D}$ if and only if $M$ is locally congruent to an open part of a tube around a totally geodesic $\mathbb{H} P^{n}$ in $G_{2}\left(\mathbb{C}^{m+2}\right)$, where $m=2 n$.

As a generalization of the well-known connection defined by Tanaka in [14] and, independently, by Webster in [16], Tanno [15] introduced the notion of generalized Tanaka Webster connection (in short, g-Tanaka-Webster connection). This connection coincides with Tanaka-Webster connection if the associated CR-structure is integrable. Here Tanaka-Webster connection was defined as the canonical affine connection on a non-degenerate, pseudo-Hermitian CRmanifold. Moreover, on real hypersurfaces in Kähler manifolds with almost contact metric structure $(\phi, \xi, \eta, g)$, the g-Tanaka-Webster connection $\hat{\nabla}^{(k)}$ for a non-zero real number $k$ was given by Cho (see [5]). In particular, if a real hypersurface satisfies $\phi A+A \phi=2 k \phi$, then the g-Tanaka-Webster connection $\hat{\nabla}^{(k)}$ coincides with the Tanaka-Webster connection.

Recently, by using the g-Tanaka-Webster connection $\hat{\nabla}^{(k)}$ Jeong, Suh and the second author have studied some parallelism of the shape operator on real hypersurfaces in complex two-plane Grassmannians ([8] and [9]). For example, 
they proved that the shape operator on a Hopf hypersurface in $G_{2}\left(\mathbb{C}^{m+2}\right)$ is $\mathfrak{D}^{\perp}$-parallel with respect to the g-Tanaka-Webster connection, that is, the shape operator $A$ of $M$ satisfies the condition $\left(\hat{\nabla}_{X}^{(k)} A\right) Y=0$ for any tangent vector fields $X \in \mathfrak{D}^{\perp}$ and $Y \in T M$ if and only if a real hypersurface in $G_{2}\left(\mathbb{C}^{m+2}\right)$ is locally congruent to an open part of a tube around a totally geodesic $\mathbb{H} P^{n}$ in $G_{2}\left(\mathbb{C}^{m+2}\right), m=2 n$ (see [9]).

In this paper, let us consider a new notion which becomes another extension for the parallelism of the shape operator on real hypersurfaces $M$ in $G_{2}\left(\mathbb{C}^{m+2}\right)$ with respect to the g-Tanaka-Webster connection. For given a tensor $T$ of type $(1,1)$ on $M$ we will say that $T$ is of Codazzi type with respect to the $g$ Tanaka-Webster connection if it satisfies $\left(\hat{\nabla}_{X}^{(k)} T\right) Y=\left(\hat{\nabla}_{Y}^{(k)} T\right) X$ for any tangent vector fields $X$ and $Y$ on $M$. By virtue of this notion we will consider a real hypersurface in $G_{2}\left(\mathbb{C}^{m+2}\right)$ whose shape operator $A$ is of Codazzi type with respect to $\hat{\nabla}^{(k)}$, that is, the shape operator $A$ of $M$ in $G_{2}\left(\mathbb{C}^{m+2}\right)$ satisfies the property

$$
\left(\hat{\nabla}_{X}^{(k)} A\right) Y=\left(\hat{\nabla}_{Y}^{(k)} A\right) X
$$

for any tangent vector fields $X$ and $Y$ on $M$. By using such the notion for the shape operator, we give a classification theorem for real hypersurfaces in $G_{2}\left(\mathbb{C}^{m+2}\right)$ as follows:

Main Theorem. There does not exist any Hopf hypersurface, $\alpha \neq 2 k$, in complex two-plane Grassmannians $G_{2}\left(\mathbb{C}^{m+2}\right), m \geq 3$, whose shape operator is of Codazzi type with respect to the generalized Tanaka-Webster connection if the distribution $\mathfrak{D}$ and $\mathfrak{D}^{\perp}$-components of the Reeb vector field are invariant by the shape operator.

Remark. In [7], the authors have remarked the case $\alpha=2 k$ ( $k$ is a nonzero real number) on Hopf hypersurfaces in $G_{2}\left(\mathbb{C}^{m+2}\right)$ with $\xi \in \mathfrak{D}^{\perp}$ (see Proposition 3.7). That is, under assumptions, $\xi \in \mathfrak{D}^{\perp}$ and $\alpha=2 k$, the shape operator $A$ of $M$ becomes naturally g-Tanaka-Webster Reeb parallel. Thus in this paper, we only consider for a Hopf hypersurface in $G_{2}\left(\mathbb{C}^{m+2}\right)$ with $\alpha \neq 2 k$, when the Reeb vector field $\xi$ belongs to $\mathfrak{D}^{\perp}$.

\section{Some fundamental formulas for real hypersurfaces in $G_{2}\left(\mathbb{C}^{m+2}\right)$ and the generalized Tanaka-Webster connection}

In this section, we first review some basic formulas and the Codazzi equation for a real hypersurface in $G_{2}\left(\mathbb{C}^{m+2}\right)$ introduced in [3], [4], [9], [12], [13], etc.

Let $M$ be a real hypersurface of $G_{2}\left(\mathbb{C}^{m+2}\right)$, that is, a submanifold of $G_{2}\left(\mathbb{C}^{m+2}\right)$ with real codimension one. The induced Riemannian metric on $M$ will also be denoted by $g$, and $\nabla$ denotes the Riemannian connection of $(M, g)$. Let $N$ be a local unit normal vector field of $M$ and $A$ the shape operator of $M$ with respect to $N$. Now let us put

$$
J X=\phi X+\eta(X) N, \quad J_{\nu} X=\phi_{\nu} X+\eta_{\nu}(X) N
$$


for any tangent vector field $X$ of a real hypersurface $M$ in $G_{2}\left(\mathbb{C}^{m+2}\right)$. From the Kähler structure $J$ of $G_{2}\left(\mathbb{C}^{m+2}\right)$ there exists an almost contact metric structure $(\phi, \xi, \eta, g)$ induced on $M$ in such a way that

$$
\phi^{2} X=-X+\eta(X) \xi, \quad \eta(\xi)=1, \quad \phi \xi=0, \quad \eta(X)=g(X, \xi)
$$

for any vector field $X$ on $M$. Furthermore, let $\left\{J_{1}, J_{2}, J_{3}\right\}$ be a canonical local basis of $\mathfrak{J}$. Then the quaternionic Kähler structure $J_{\nu}$ of $G_{2}\left(\mathbb{C}^{m+2}\right)$, together with the condition $J_{\nu} J_{\nu+1}=J_{\nu+2}=-J_{\nu+1} J_{\nu}$, induces an almost contact metric 3-structure $\left(\phi_{\nu}, \xi_{\nu}, \eta_{\nu}, g\right)$ on $M$ as follows :

$$
\begin{aligned}
& \phi_{\nu}^{2} X=-X+\eta_{\nu}(X) \xi_{\nu}, \quad \eta_{\nu}\left(\xi_{\nu}\right)=1, \quad \phi_{\nu} \xi_{\nu}=0, \\
& \phi_{\nu+1} \xi_{\nu}=-\xi_{\nu+2}, \quad \phi_{\nu} \xi_{\nu+1}=\xi_{\nu+2}, \\
& \phi_{\nu} \phi_{\nu+1} X=\phi_{\nu+2} X+\eta_{\nu+1}(X) \xi_{\nu}, \\
& \phi_{\nu+1} \phi_{\nu} X=-\phi_{\nu+2} X+\eta_{\nu}(X) \xi_{\nu+1}
\end{aligned}
$$

for any vector field $X$ tangent to $M$. Moreover, from the commuting property of $J_{\nu} J=J J_{\nu}, \nu=1,2,3$ and (1.1), the relation between these two contact metric structures $(\phi, \xi, \eta, g)$ and $\left(\phi_{\nu}, \xi_{\nu}, \eta_{\nu}, g\right), \nu=1,2,3$, can be given by

$$
\begin{aligned}
& \phi \phi_{\nu} X=\phi_{\nu} \phi X+\eta_{\nu}(X) \xi-\eta(X) \xi_{\nu}, \\
& \eta_{\nu}(\phi X)=\eta\left(\phi_{\nu} X\right), \quad \phi \xi_{\nu}=\phi_{\nu} \xi .
\end{aligned}
$$

On the other hand, as $J$ is a Kähler structure (i.e., $\tilde{\nabla} J=0$ ) and $\mathfrak{J}$ a quaternionic Kähler structure (i.e., $\tilde{\nabla}_{X} J_{\nu}=q_{\nu+2}(X) J_{\nu+1}-q_{\nu+1}(X) J_{\nu+2}$ for any tangent vector fields $X$ on $\left.G_{2}\left(\mathbb{C}^{m+2}\right)\right)$, together with Gauss and Weingarten formulas it follows that

$$
\begin{gathered}
\left(\nabla_{X} \phi\right) Y=\eta(Y) A X-g(A X, Y) \xi, \quad \nabla_{X} \xi=\phi A X \\
\nabla_{X} \xi_{\nu}=q_{\nu+2}(X) \xi_{\nu+1}-q_{\nu+1}(X) \xi_{\nu+2}+\phi_{\nu} A X \\
\left(\nabla_{X} \phi_{\nu}\right) Y=-q_{\nu+1}(X) \phi_{\nu+2} Y+q_{\nu+2}(X) \phi_{\nu+1} Y \\
+\eta_{\nu}(Y) A X-g(A X, Y) \xi_{\nu}
\end{gathered}
$$

Using the expression for the curvature tensor $\tilde{R}$ of $G_{2}\left(\mathbb{C}^{m+2}\right)$ in [9], the equation of Codazzi is given by

$$
\begin{aligned}
\left(\nabla_{X} A\right) Y-\left(\nabla_{Y} A\right) X= & \eta(X) \phi Y-\eta(Y) \phi X-2 g(\phi X, Y) \xi \\
& +\sum_{\nu=1}^{3}\left\{\eta_{\nu}(X) \phi_{\nu} Y-\eta_{\nu}(Y) \phi_{\nu} X-2 g\left(\phi_{\nu} X, Y\right) \xi_{\nu}\right\} \\
& +\sum_{\nu=1}^{3}\left\{\eta_{\nu}(\phi X) \phi_{\nu} \phi Y-\eta_{\nu}(\phi Y) \phi_{\nu} \phi X\right\} \\
& +\sum_{\nu=1}^{3}\left\{\eta(X) \eta_{\nu}(\phi Y)-\eta(Y) \eta_{\nu}(\phi X)\right\} \xi_{\nu}
\end{aligned}
$$


As mentioned in Theorem A, the complete classification of real hypersurfaces in $G_{2}\left(\mathbb{C}^{m+2}\right), m \geq 3$, with two kinds of $A$-invariancy for the distributions $[\xi]=\operatorname{Span}\{\xi\}$ and $\mathfrak{D}^{\perp}=\operatorname{Span}\left\{\xi_{1}, \xi_{2}, \xi_{3}\right\}$ was obtained by Berndt and Suh [3]. Accordingly we introduce the following two propositions related to the principal curvatures of the model spaces (A) and (B), respectively.

Proposition A. Let $M$ be a connected real hypersurface of $G_{2}\left(\mathbb{C}^{m+2}\right)$. Suppose that $A \mathfrak{D} \subset \mathfrak{D}, A \xi=\alpha \xi$, and $\xi$ is tangent to $\mathfrak{D}^{\perp}$. Let $J_{1} \in \mathfrak{J}$ be the almost Hermitian structure such that $J N=J_{1} N$. Then $M$ has the following three (if $r=\pi / 2 \sqrt{8}$ ) or four (otherwise) distinct constant principal curvatures:

\begin{tabular}{l|c|l}
\hline principal curvature & multiplicity & \multicolumn{1}{c}{ eigenspace } \\
\hline$\alpha=\sqrt{8} \cot (\sqrt{8} r)$ & 1 & $T_{\alpha}=\mathbb{R} \xi=\mathbb{R} J N=\mathbb{R} \xi_{1}=\operatorname{Span}\{\xi\}$ \\
$\beta=\sqrt{2} \cot (\sqrt{2} r)$ & 2 & $T_{\beta}=\mathbb{C}^{\perp} \xi=\operatorname{Span}\left\{\xi_{2}, \xi_{3}\right\}$ \\
$\lambda=-\sqrt{2} \tan (\sqrt{2} r)$ & $2(m-1)$ & $T_{\lambda}=\left\{X \mid X \perp \mathbb{H} \xi, J X=J_{1} X\right\}$ \\
$\mu=0$ & $2(m-1)$ & $T_{\mu}=\left\{X \mid X \perp \mathbb{H} \xi, J X=-J_{1} X\right\}$ \\
\hline
\end{tabular}

with some $r \in(0, \pi / \sqrt{8})$. Here $\mathbb{R} \xi, \mathbb{C} \xi$ and $\mathbb{H} \xi$ respectively denotes real, complex and quaternionic span of the structure vector field $\xi$ and $\mathbb{C}^{\perp} \xi$ denotes the orthogonal complement of $\mathbb{C} \xi$ in $\mathbb{H} \xi$.

Proposition B. Let $M$ be a connected real hypersurface of $G_{2}\left(\mathbb{C}^{m+2}\right)$. Suppose that $A \mathfrak{D} \in \mathfrak{D}, A \xi=\alpha \xi$, and $\xi$ is tangent to $\mathfrak{D}$. Then the quaternionic dimension $m$ of $G_{2}\left(\mathbb{C}^{m+2}\right)$ is even, say $m=2 n$, and $M$ has five distinct constant principal curvatures and the corresponding multiplicities with respect to the eigenspaces:

\begin{tabular}{l|c|l}
\hline principal curvature & multiplicity & \multicolumn{1}{c}{ eigenspace } \\
\hline$\alpha=-2 \tan (2 r)$ & 1 & $T_{\alpha}=\operatorname{Span}\{\xi\}$ \\
$\beta=2 \cot (2 r)$ & 3 & $T_{\beta}=\operatorname{Span}\left\{\xi_{\nu} \mid \nu=1,2,3\right\}$ \\
$\gamma=0$ & 3 & $T_{\gamma}=\operatorname{Span}\left\{\phi_{\nu} \xi \mid \nu=1,2,3\right\}$ \\
$\lambda=\cot (r)$ & $4 n-4$ & $T_{\lambda}$ \\
$\mu=-\tan (r)$ & $4 n-4$ & $T_{\mu}$ \\
\hline
\end{tabular}

where $r \in(0, \pi / \sqrt{8})$ and $T_{\lambda} \oplus T_{\mu}=(\mathbb{H} \mathbb{C} \xi)^{\perp}, \mathfrak{J} T_{\lambda}=T_{\lambda}, \mathfrak{J} T_{\mu}=T_{\mu}, J T_{\lambda}=T_{\mu}$.

From now on, we introduce the generalized Tanaka-Webster (shortly, gTanaka-Webster) connection for a real hypersurface in Kähler manifolds (see [5], [6], [8], [9] and [10]).

As stated in the introduction, the Tanaka-Webster connection is the canonical affine connection defined on a non-degenerate pseudo-Hermitian CR-manifold (see [14] and [16]). Tanno [15] defined the g-Tanaka-Webster connection for contact metric manifolds by the canonical connection as follows :

$$
\hat{\nabla}_{X} Y=\nabla_{X} Y+\left(\nabla_{X} \eta\right)(Y) \xi-\eta(Y) \nabla_{X} \xi-\eta(X) \phi Y
$$


for any vector fields $X$ and $Y$. It coincides with the Tanaka-Webster connection if the associated CR-structure is integrable.

On the other hand, a real hypersurface $M$ in Kähler manifolds has the property $A \phi+\phi A= \pm 2 \phi$ (the sign depends on the orientation) for the shape operator $A$ and the structure tensor $\phi$ of $M$ if and only if the almost contact metric structure of $M$ is contact metric (see [5]). From such a point of view, Cho defined the generalized Tanaka-Webster connection $\hat{\nabla}^{(k)}$ on $M$ by the naturally extended one of Tanno's g-Tanaka-Webster connection $\hat{\nabla}$ for contact metric manifolds:

$$
\hat{\nabla}_{X}^{(k)} Y=\nabla_{X} Y+g(\phi A X, Y) \xi-\eta(Y) \phi A X-k \eta(X) \phi Y
$$

for a non-zero real number $k$. Moreover, if a real hypersurface in Kähler manifolds satisfies $A \phi+\phi A=2 k \phi$, then the associated CR-structure is pseudohermitian, strongly pseudo-convex, integrable. Thus under the assumption $A \phi+\phi A=2 k \phi$, we obtain the following facts (see [5], [8] and [10]) :

(1) if $k=1$, then we see that $\hat{\nabla}^{(k)}=\hat{\nabla}$,

(2) the connection $\hat{\nabla}^{(k)}$ coincides with the Tanaka-Webster connection defined as the canonical affine connection on a non-degenerate, pseudoHermitian CR-manifold.

Thus we see that the connection $\hat{\nabla}^{(k)}$ is a natural generalization of TanakaWebster connection and call it generalized Tanaka-Webster connection (or shortly, g-Tanaka-Webster connection) on real hypersurfaces of Kähler manifolds.

\section{Proof of Main Theorem}

First of all, we will prove that the Reeb vector field $\xi$ belongs to either the distribution $\mathfrak{D}$ or the distribution $\mathfrak{D}^{\perp}$ for a Hopf hypersurface $M$ in $G_{2}\left(\mathbb{C}^{m+2}\right)$ whose shape operator is of Codazzi type with respect to the generalized TanakaWebster connection $\hat{\nabla}^{(k)}$.

Lemma 2.1. Let $M$ be a Hopf hypersurface in $G_{2}\left(\mathbb{C}^{m+2}\right), m \geq 3$, whose shape operator is of Codazzi type with respect to the generalized Tanaka-Webster connection. If $\mathfrak{D}$ and $\mathfrak{D}^{\perp}$-components of $\xi$ are invariant under the shape operator of $M$, then the principal curvature $\alpha=g(A \xi, \xi)$ is constant. Furthermore, the Reeb vector field $\xi$ belongs to either the distribution $\mathfrak{D}$ or the distribution $\mathfrak{D}^{\perp}$.

Proof. To show this fact, we assume that the Reeb vector field $\xi$ satisfies

$$
\xi=\eta\left(X_{0}\right) X_{0}+\eta\left(\xi_{1}\right) \xi_{1} \text { such that } \eta\left(X_{0}\right) \eta\left(\xi_{1}\right) \neq 0
$$

for some unit vectors $X_{0} \in \mathfrak{D}$ and $\xi_{1} \in \mathfrak{D}^{\perp}$. 
Let us consider the case that the smooth function $\alpha=g(A \xi, \xi)$ vanishes. In [3] Berndt and Suh gave

$$
Y \alpha=(\xi \alpha) \eta(Y)-4 \sum_{\nu=1}^{3} \eta_{\nu}(\xi) \eta_{\nu}(\phi Y)
$$

for any tangent vector field $Y$ on $M$ under the assumption that $M$ is Hopf. Now, as $\alpha=0$, it implies that

$$
\sum_{\nu=1}^{3} \eta_{\nu}(\xi) \phi_{\nu} \xi=0
$$

Moreover, using the equation $(* *)$, we have $\eta\left(X_{0}\right) \phi X_{0}=0$. Since $\eta\left(X_{0}\right) \neq 0$, the tangent vector $\phi X_{0} \in T_{x} M$ becomes a zero one. This gives a contradiction and we see that $\xi$ belongs to either $\mathfrak{D}$ or $\mathfrak{D}^{\perp}$ when $\alpha$ is vanishing. In addition, from the equation $(2.1)$ we see that if $\alpha$ is constant, then $\xi$ belongs to either $\mathfrak{D}$ or $\mathfrak{D}^{\perp}$.

From now on, let us consider the case $\alpha \neq 0$. Since the shape operator $A$ of $M$ satisfies the Codazzi type equation for $\hat{\nabla}^{(k)}$, that is, $\left(\hat{\nabla}_{X}^{(k)} A\right) Y=\left(\hat{\nabla}_{Y}^{(k)} A\right) X$ for any tangent vector fields $X, Y$ on $M$, we have

$$
\begin{aligned}
& \left(\nabla_{X} A\right) Y+g(\phi A X, A Y) \xi-\eta(A Y) \phi A X-k \eta(X) \phi A Y \\
& -g(\phi A X, Y) A \xi+\eta(Y) A \phi A X+k \eta(X) A \phi Y \\
= & \left(\nabla_{Y} A\right) X+g(\phi A Y, A X) \xi-\eta(A X) \phi A Y-k \eta(Y) \phi A X \\
& -g(\phi A Y, X) A \xi+\eta(X) A \phi A Y+k \eta(Y) A \phi X,
\end{aligned}
$$

together with the equation (1.8). Moreover, it can be written as

$$
\begin{aligned}
& \eta(X) \phi Y-\eta(Y) \phi X-2 g(\phi X, Y) \xi \\
& +2 g(A \phi A X, Y) \xi-\alpha \eta(Y) \phi A X-k \eta(X) \phi A Y-\alpha g(\phi A X, Y) \xi \\
& +\eta(Y) A \phi A X+k \eta(X) A \phi Y+\alpha \eta(X) \phi A Y+k \eta(Y) \phi A X \\
& -\alpha g(A \phi X, Y) \xi-\eta(X) A \phi A Y-k \eta(Y) A \phi X \\
& +\sum_{\nu=1}^{3}\left\{\eta_{\nu}(X) \phi_{\nu} Y-\eta_{\nu}(Y) \phi_{\nu} X-2 g\left(\phi_{\nu} X, Y\right) \xi_{\nu}+\eta_{\nu}(\phi X) \phi_{\nu} \phi Y\right. \\
& \left.\quad-\eta_{\nu}(\phi Y) \phi_{\nu} \phi X+\eta(X) \eta_{\nu}(\phi Y) \xi_{\nu}-\eta(Y) \eta_{\nu}(\phi X) \xi_{\nu}\right\}=0
\end{aligned}
$$

using the assumption $A \xi=\alpha \xi$ and the Codazzi equation (1.7).

Putting $Y$ by $\xi$ in $(2.2)$, we get

$$
\begin{aligned}
& -\phi X+\sum_{\nu=1}^{3}\left\{\eta_{\nu}(X) \phi_{\nu} \xi-\eta_{\nu}(\xi) \phi_{\nu} X-3 g\left(\phi_{\nu} X, \xi\right) \xi_{\nu}\right\} \\
& -\alpha \phi A X+A \phi A X+k \phi A X-k A \phi X=0
\end{aligned}
$$

for any vector field $X$ tangent on $M$. 
Substituting $X=X_{0}$ in (2.3) and using (**), we have

$$
\phi X_{0}+\eta\left(\xi_{1}\right) \phi_{1} X_{0}+\alpha \phi A X_{0}-A \phi A X_{0}-k \phi A X_{0}+k A \phi X_{0}=0 .
$$

Since $\phi \xi=0$ and $(* *)$, we see that $\phi X_{0}=-\eta\left(\xi_{1}\right) \phi_{1} X_{0}$. From this, the equation (2.4) is written as

$$
\alpha \phi A X_{0}-A \phi A X_{0}-k \phi A X_{0}+k A \phi X_{0}=0 .
$$

On the other hand, from our assumptions that the $\mathfrak{D}$ and $\mathfrak{D}^{\perp}$-components of $\xi$ are invariant under the shape operator of $M$ and $M$ is Hopf, we see that the unit vector field $X_{0} \in \mathfrak{D}$ becomes a principal one, $A X_{0}=\alpha X_{0}$. Thus (2.5) changes to

$$
\alpha^{2} \phi X_{0}-\alpha A \phi X_{0}-k \alpha \phi X_{0}+k A \phi X_{0}=0 .
$$

In addition, if the $\mathfrak{D}$-component of $\xi$ is a principal vector field with the corresponding principal curvature $\alpha(\neq 0)$, then $\phi X_{0}$ is also principal vector field, that is, $A \phi X_{0}=\left(\left(\alpha^{2}+4 \eta^{2}\left(X_{0}\right)\right) / \alpha\right) \phi X_{0}$ (see Proposition 2 in [4]). Thus we have

$$
\alpha^{2} \phi X_{0}-\left(\alpha^{2}+4 \eta^{2}\left(X_{0}\right)\right) \phi X_{0}-k \alpha \phi X_{0}+\frac{k\left(\alpha^{2}+4 \eta^{2}\left(X_{0}\right)\right)}{\alpha} \phi X_{0}=0
$$

it follows that $4 \eta^{2}\left(X_{0}\right)(k-\alpha) \phi X_{0}=0$. Taking the inner product with $\phi X_{0}$, we obtain $-4 \eta^{2}\left(X_{0}\right) \eta^{2}\left(\xi_{1}\right)(k-\alpha)=0$. Since $\eta\left(X_{0}\right) \eta\left(\xi_{1}\right) \neq 0$, we have $\alpha=k$. It implies that the smooth function $\alpha$ is constant. By virtue of the proof for the case $\alpha=0$, the Reeb vector field $\xi$ belongs to either the distribution $\mathfrak{D}$ or the distribution $\mathfrak{D}^{\perp}$.

From Lemma 2.1, we can consider the following two cases:

- The Reeb vector field $\xi$ belongs to distribution $\mathfrak{D}^{\perp}$, that is, $\xi \in \mathfrak{D}^{\perp}$,

- $\xi$ belongs to $\mathfrak{D}$, that is, $\xi \in \mathfrak{D}$.

Now, we assume that $\xi \in \mathfrak{D}^{\perp}$. For the sake of convenience we put $\xi=\xi_{1}$.

Lemma 2.2. Let $M$ be a Hopf hypersurface, $\alpha \neq 2 k$, in $G_{2}\left(\mathbb{C}^{m+2}\right), m \geq 3$, whose shape operator is of Codazzi type in generalized Tanaka-Webster connection. If the Reeb vector field $\xi$ belongs to the distribution $\mathfrak{D}^{\perp}$, then the shape operator $A$ commutes with the structure tensor $\phi$.

Proof. Using $\xi=\xi_{1}$ and (2.3), we have

$$
\begin{aligned}
& -\phi X+\left\{\eta_{2}(X) \phi_{2} \xi+\eta_{3}(X) \phi_{3} \xi-\phi_{1} X-3 g\left(\phi_{2} X, \xi\right) \xi_{2}-3 g\left(\phi_{3} X, \xi\right) \xi_{3}\right\} \\
& -\alpha \phi A X+A \phi A X+k \phi A X-k A \phi X=0
\end{aligned}
$$

that is,

(2.6) $-\phi X-\phi_{1} X+2 \eta_{2}(X) \xi_{3}-2 \eta_{3}(X) \xi_{2}-\alpha \phi A X+A \phi A X+k \phi A X-k A \phi X=0$

for any tangent vector field $X$ on $M$. 
Now we introduce the formula derived from $A \xi=\alpha \xi$ (see [4]) as follows:

$$
\begin{aligned}
& \alpha A \phi X+\alpha \phi A X-2 A \phi A X+2 \phi X \\
= & -2 \sum_{\nu=1}^{3}\left\{\eta_{\nu}(X) \phi_{\nu} \xi+\eta_{\nu}(\phi X) \xi_{\nu}+\eta_{\nu}(\xi) \phi_{\nu} X\right. \\
& \left.-2 \eta(X) \eta_{\nu}(\xi) \phi_{\nu} \xi-2 \eta_{\nu}(\phi X) \eta_{\nu}(\xi) \xi\right\} .
\end{aligned}
$$

Combining above equations, we have

$$
\begin{aligned}
& \phi X+\phi_{1} X-2 \eta_{2}(X) \xi_{3}+2 \eta_{3}(X) \xi_{2}+\alpha \phi A X-k \phi A X+k A \phi X \\
= & A \phi A X \\
= & \frac{\alpha}{2} A \phi X+\frac{\alpha}{2} \phi A X+\phi X \\
& +\sum_{\nu=1}^{3}\left\{\eta_{\nu}(X) \phi_{\nu} \xi+\eta_{\nu}(\phi X) \xi_{\nu}+\eta_{\nu}(\xi) \phi_{\nu} X\right. \\
& \left.\quad-2 \eta(X) \eta_{\nu}(\xi) \phi_{\nu} \xi-2 \eta_{\nu}(\phi X) \eta_{\nu}(\xi) \xi\right\} \\
= & \frac{\alpha}{2} A \phi X+\frac{\alpha}{2} \phi A X+\phi X \\
& +\left\{\eta_{2}(X) \phi_{2} \xi+\eta_{3}(X) \phi_{3} \xi+\eta_{2}(\phi X) \xi_{2}+\eta_{3}(\phi X) \xi_{3}+\phi_{1} X\right\} \\
= & \frac{\alpha}{2} A \phi X+\frac{\alpha}{2} \phi A X+\phi X \\
& -\eta_{2}(X) \xi_{3}+\eta_{3}(X) \xi_{2}+\eta_{3}(X) \xi_{2}-\eta_{2}(X) \xi_{3}+\phi_{1} X .
\end{aligned}
$$

Therefore, we get

$$
\left(\frac{\alpha}{2}-k\right) \phi A X-\left(\frac{\alpha}{2}-k\right) A \phi X=0
$$

that is,

$$
\left(\frac{\alpha}{2}-k\right)(\phi A-A \phi) X=0
$$

Since $\alpha \neq 2 k$, we obtain $(\phi A-A \phi) X=0$ for any tangent vector field $X$ on $M$. It means that the shape operator $A$ commutes with the structure tensor $\phi$.

Due to Berdnt and Suh [4], the Reeb flow on $M$ is isometric if and only if the structure tensor field $\phi$ commutes with the shape operator $A$ of $M$, that is, $A \phi=\phi A$. Thus, from Lemma 2.2 and Theorem B, we conclude that $M$ is of type (A) under our assumptions.

Conversely, let $M$ be a real hypersurface of type (A) in $G_{2}\left(\mathbb{C}^{m+2}\right)$ and check if the shape operator $A$ of $M$ satisfies the Codazzi type equation (*) for the $\mathrm{g}$-Tanaka-Webster connection. In order to do this, we suppose that the shape operator $A$ of $M$ satisfies (*). 
Putting $X \in T_{\lambda}$ and $Y \in T_{\mu}$ in (2.2), we get

$$
\begin{aligned}
0 & =-2 \sum_{\nu=1}^{3} g\left(\phi_{\nu} X, Y\right) \xi_{\nu} \\
& =-2 g\left(\phi_{1} X, Y\right) \xi_{1}-2 g\left(\phi_{2} X, Y\right) \xi_{2}-2 g\left(\phi_{3} X, Y\right) \xi_{3} .
\end{aligned}
$$

Taking the inner product with $\xi_{2}$, we have $g\left(\phi_{2} X, Y\right)=0$ for any tangent vector fields $X \in T_{\lambda}$ and $Y \in T_{\mu}$. Since $\phi_{2} X \in T_{\mu}$ for any tangent vector field $X \in T_{\lambda}$, it follows that $g\left(\phi_{2} X, \phi_{2} X\right)=0$, which means that $\phi_{2} X$ becomes a zero vector field. This gives a contradiction. So we assert that the shape operator of a real hypersurface of type $(\mathrm{A})$ in $G_{2}\left(\mathbb{C}^{m+2}\right)$ does not satisfy the Codazzi type equation $(*)$ with respect to the generalized Tanaka-Webster connection.

Next, we consider the case that the Reeb vector field $\xi$ belongs to the distribution $\mathfrak{D}$. In fact, under this assumption we conclude that a Hopf hypersurface $M$ satisfying $(*)$ is locally congruent to a model space of $(B)$ by virtue of Theorem $\mathrm{C}$. Hence, to prove our main theorem, we may check the condition $(*)$ for the real hypersurface of type $(\mathrm{B})$ in $G_{2}\left(\mathbb{C}^{m+2}\right)$.

By putting $Y=\xi$ in (2.2), we already obtain the equation (2.3). As setting $X=\xi_{1}$ in $(2.3)$, we have

$$
\begin{aligned}
& -\phi \xi_{1}+\sum_{\nu=1}^{3}\left\{\eta_{\nu}\left(\xi_{1}\right) \phi_{\nu} \xi-\eta_{\nu}(\xi) \phi_{\nu} \xi_{1}-3 g\left(\phi_{\nu} \xi_{1}, \xi\right) \xi_{\nu}\right\} \\
& -\alpha \phi A \xi_{1}+A \phi A \xi_{1}+k \phi A \xi_{1}-k A \phi \xi_{1}=0 .
\end{aligned}
$$

Since $A \xi_{1}=\beta \xi_{1}$ (see Proposition B), we get

$$
-\phi \xi_{1}+\phi_{1} \xi-\alpha \beta \phi \xi_{1}+\beta A \phi \xi_{1}+k \beta \phi \xi_{1}-k A \phi \xi_{1}=0,
$$

that is,

$$
\beta(\alpha-k) \phi \xi_{1}=0 .
$$

For some $r \in(0, \pi / 4)$, the principal curvature $\beta$ can not equal to 0 . So, we know that $\alpha=k$ where $k$ is non-zero constant. Substituting $\alpha=k$ into (2.3), we have

$$
-\phi X+\sum_{\nu=1}^{3}\left\{\eta_{\nu}(X) \phi_{\nu} \xi-\eta_{\nu}(\xi) \phi_{\nu} X-3 g\left(\phi_{\nu} X, \xi\right) \xi_{\nu}\right\}+A \phi A X-k A \phi X=0
$$

for any tangent vector field $X$ on $M$.

By putting $X \in T_{\lambda}$ in (2.8), we get $(-\lambda \mu+\alpha \mu+1) \phi X=0$, because if $X \in T_{\lambda}$, then $X$ is orthogonal to $\xi_{\nu}$ and $\phi \xi_{\nu}$ for $\nu=1,2,3$. Moreover, we see that $\phi T_{\lambda} \subset T_{\mu}$ for $X \in T_{\lambda}$. From this, we see that $\lambda \mu-\alpha \mu-1=0$. By using the properties in Proposition B, we have $1+\tan ^{2} r=0$ for some $r \in(0, \pi / 4)$. This gives a contradiction.

Hence summing up these discussions, we give a complete proof of our Main Theorem in the introduction. 
Acknowledgement. The authors would like to express their sincere gratitude to Professors Juan de Dios Pérez and Young Jin Suh for their continuous encouragement and many pieces of expert advice to develop our works and also to the referee for his/her valuable suggestions to improve the first version of our manuscript.

\section{References}

[1] D. V. Alekseevskii, Compact quaternion spaces, Funct. Anal. Appl. 2 (1968), 106-114.

[2] J. Berndt, Riemannian geometry of complex two-plane Grassmannian, Rend. Semin. Mat. Univ. Politec. Torino 55 (1997), no. 1, 19-83.

[3] J. Berndt and Y. J. Suh, Real hypersurfaces in complex two-plane Grassmannians, Monatsh. Math. 127 (1999), no. 1, 1-14.

[4] _ Real hypersurfaces with isometric Reeb flow in complex two-plane Grassmannians, Monatsh. Math. 137 (2002), no. 2, 87-98.

[5] J. T. Cho, CR structures on real hypersurfaces of a complex space form, Publ. Math. Debrecen 54 (1999), no. 3-4, 473-487.

[6] J. T. Cho and M. Kon, The Tanaka-Webster connection and real hypersurfaces in a complex space form, Kodai Math. J. 34 (2011), 474-484.

[7] I. Jeong, M. Kimura, H. Lee, and Y. J. Suh, Real hypersurfaces in complex twoplane Grassmannians with generalized Tanaka-Webster Reeb parallel shape operator, Monatsh. Math. 171 (2013), no. 3-4, 357-376.

[8] I. Jeong, H. Lee, and Y. J. Suh, Real hypersurfaces in complex two-plane Grassmannians with generalized Tanaka-Webster parallel shape operator, Kodai Math. J. 34 (2011), no. 2, 352-366.

[9] _ Real hypersurfaces in complex two-plane Grassmannians with generalized Tanaka-Webster $\mathfrak{D}^{\perp}$-parallel shape operator, Int. J. Geom. Methods Mod. Phys. 9 (2012), no. 4, 1250032, 20 pp.

[10] M. Kon, Real hypersurfaces in complex space forms and the generalized-Tanaka-Webster connection, Proceedings of the 13th International Workshop on Differential Geometry and Related Fields [Vol. 13], 145-159, Natl. Inst. Math. Sci. (NIMS), Daejeon 2009.

[11] H. Lee and Y. J. Suh, Real hypersurfaces of type B in complex two-plane Grassmannians related to the Reeb vector, Bull. Korean Math. Soc. 47 (2010), no. 3, 551-561.

[12] J. D. Pérez and Y. J. Suh, The Ricci tensor of real hypersurfaces in complex two-plane Grassmannians, J. Korean Math. Soc. 44 (2007), no. 1, 211-235.

[13] Y. J. Suh, Real hypersurfaces in complex two-plane Grassmannians with parallel shape operator, Bull. Aust. Math. Soc. 67 (2003), no. 3, 493-502.

[14] N. Tanaka, On non-degenerate real hypersurfaces, graded Lie algebras and Cartan connections, Jpn. J. Math. 20 (1976), no. 1, 131-190.

[15] S. Tanno, Variational problems on contact Riemannian manifolds, Trans. Amer. Math. Soc. 314 (1989), no. 1, 349-379.

[16] S. M. Webster, Pseudo-Hermitian structures on a real hypersurface, J. Differential Geom. 13 (1978), no. 1, 25-41.

Kyusuk CHO

Department of Mathematics

KyUngPoOK NATIONAL University

DAEGU 702-701, Korea

E-mail address: choks4101@nate.com 
HyUnJin LEE

The Center for Geometry and its Applications

Pohang University of Science \& Technology

Pohang 790-784, Korea

E-mail address: 1hjibis@hanmail.net

EUNMI PAK

Department of Mathematics

KYUNGPOOK NATIONAL UNIVERSITY

DAEGU 702-701, KorEA

E-mail address: empak@knu.ac.kr 